\title{
镍催化均相不对称氢化反应研究进展
}

\author{
刘元华 $a$ 董秀琴*, $a$ 张绪穆 ${ }^{b}$ \\ ( ${ }^{a}$ 武汉大学化学与分子科学学院 生物医用高分子材料教育部重点实验室 \\ 有机硅化合物及材料教育部工程中心 武汉 430072) \\ ( ${ }^{b}$ 南方科技大学化学系 广东深圳 518055)
}

\begin{abstract}
摘要 过渡金属络合物催化的均相不对称氢化反应是合成手性化合物的重要方法之一，目前主要集中于钉、铑、铱和 钯等贵重过渡金属催化体系，这些贵重过渡金属催化体系面临着地球储量有限、价格昂贵和重金属污染环境等问题， 因而发展地球储量丰富、价格低廉、无毒或低毒且对环境友好的铁、钴、镍和铜的均相不对称氢化反应催化体系符合 现代化学可持续发展的要求和趋势. 简要综述了近些年来廉价金属镍催化的均相不对称氢化反应研究领域的新进展, 基于前手性不饱和化合物双键的不同类型，即碳-氧双键 $(\mathrm{C}=\mathrm{O})$ 、碳-碳双键 $(\mathrm{C}=\mathrm{C})$ 以及碳-氮双键 $(\mathrm{C}=\mathrm{N})$ 等，依次介绍 它们的研究现状, 目前已经取得了突破和可观的研究成果, 系统地分析了镍催化体系中催化氢化不同类型底物的优势 与不足, 并展望了未来的研究方向.
\end{abstract}

关键词 不对称氢化; 镍催化; 手性双膦配体; 手性化合物; 立体选择性

\section{Recent Advances of Nickel-Catalyzed Homogeneous Asymmetric Hydrogenation}

\author{
Liu, Yuanhua $^{a}$ Dong, Xiu-Qin*,a ${ }^{*}$ Zhang, Xumu ${ }^{b}$ \\ ( ${ }^{a}$ Key Laboratory of Biomedical Polymers, Engineering Research Center of Organosilicon Compounds \& Materials, Ministry \\ of Education, College of Chemistry and Molecular Sciences, Wuhan University, Wuhan 430072) \\ $\left({ }^{b}\right.$ Department of Chemistry, Southern University of Science and Technology, Shenzhen, Guangdong 518055)
}

\begin{abstract}
Transition metal complexes-catalyzed homogeneous asymmetric hydrogenation is an important method for the synthesis of chiral compounds. At present, it is mainly focused on precious transition metal catalytic systems, such as ruthenium, rhodium, iridium and palladium. However, they are suffered from the difficulties of limited resource, high cost and environmental contamination. Therefore, it is important and necessary to develop catalytic systems based on cheap, non-toxic or low toxic, environmentally friendly and earth-abundant iron, cobalt, nickel, copper transition metal, which are in accordance with the requirements and research trend of the sustainable development of modern chemistry. The recent progress of nickel-catalyzed homogeneous asymmetric hydrogenation of prochiral unsaturated compounds containing carbon oxygen double bond $(\mathrm{C}=\mathrm{O})$, carbon carbon double bond $(\mathrm{C}=\mathrm{C})$ and carbon nitrogen double bond $(\mathrm{C}=\mathrm{N})$ is reviewed, and some breakthroughs and considerable research results achieved are introduced. In addition, the advantages and disadvantages of different types of substrates in nickel catalyst system are analyzed, and the future research direction is prospected.
\end{abstract}

Keywords asymmetric hydrogenation; nickel catalysis; chiral diphosphine ligand; chiral compounds; stereoselectivity

手性过渡金属络合物催化的均相不对称氢化含有 碳一碳双键 $(C=C)$ 、碳一氧双键 $(C=O)$ 以及碳一氮双键 $(C=N)$ 等不饱和键的前手性化合物是目前构建手性化 合物的重要方法之一，该方法具有直接、高效和高原子 经济性等优势, 被广泛应用于构建手性醇、手性胺、手
性羧酸和手性氨基酸等多种类型的手性分子 ${ }^{[1]}$. 目前, 均相不对称催化氢化反应体系主要是基于钉、铑、铱和 钯等贵重过渡金属 ${ }^{[1]}$, 取得了十分瞩目的成就，这些贵 重过渡金属催化体系存在着地球储量有限、价格昂贵、 重金属污染环境等问题，因而发展地球储量丰富、价格

\footnotetext{
* Corresponding author. E-mail: xiuqindong@whu.edu.cn

Received December 19, 2019; revised January 20, 2020; published online February 14, 2020.

Project supported by the National Natural Science Foundation of China (No. 21502145), the Wuhan Morning Light Plan of Youth Science and Technology (No. 2017050300307) and the National Natural Science Foundation of Jiangsu Province (No. SBK2019041078).

国家自然科学基金(No. 21502145)、武汉市青年科技晨光计划(No. 2017050300307)和江苏省自然科学基金(No. SBK2019041078)资助项目.
} 
低廉、无毒或低毒且对环境友好的符合现代化学可持续 发展要求的铁、钴、镍和铜的均相不对称氢化催化体系 显得非常的重要 ${ }^{[2]}$. 镍作为地壳中蕴藏量最丰富的金属 元素之一, 具有价格便宜、储量丰富、环境友好等优点, 用金属镍来替代钉、铑、铱和钯等贵重金属进行催化不 对称氢化不饱和化合物非常具有吸引力, 是不对称催化 氢化一个新的研究热点, 近些年来备受关注. 目前, 虽 然镍催化的还原反应报道比较多 ${ }^{[3]}$, 但是关于镍催化的 均相不对称氢化反应却鲜有报道, 主要是由于一方面需 要解决催化活性问题, 与此同时需要控制好反应的立体 选择性, 另一方面人们对其催化机理的认识有所欠缺, 从而导致了镍催化的均相不对称氢化反应的进展相对 比较缓慢. 直到 2016 年 Chirik 等 ${ }^{[4]}$ 对镍催化 $\alpha, \beta$-不饱和 羧酸酯的均相不对称氢化反应的催化机理进行了深入 研究, 为后续发展的镍催化的均相不对称氢化反应起到 了重要的指导作用, 使得后续镍催化的不对称氢化反应 较为迅猛的发展. 因此, 我们系统地介绍和整理了近年 来镍催化的均相不对称氢化反应的研究现状和取得的 一些进展, 希望有助于深入认识和理解镍催化的均相不 对称氢化反应, 并为后续有关领域的研究提供一定的探 索和指示. 本文将按照前手性不饱和化合物的双键类型 依次进行介绍, 包括碳-氧双键 $(\mathrm{C}=\mathrm{O})$ 、碳-碳双键 $(\mathrm{C}=$ $C)$ 以及碳一氮双键 $(C=N)$ 等.

\section{1 镍催化的前手性酮(C=0 键)均相不对称氢 化反应}

到目前为止, 关于镍催化前手性不饱和酮 $(C=O$ 键)的均相不对称氢化反应的报道甚少. 2008 年, Hamada 等 ${ }^{[5]}$ 采用 $\mathrm{Ni}(\mathrm{OAc})_{2} \bullet \mathrm{H}_{2} \mathrm{O} / \mathrm{Josiphos}$ 类型的配体 $\mathbf{L 1}$ 的催化 体系, 通过动态动力学拆分策略首次实现了 $\alpha$-氨基 $-\beta$ 羰基羧酸酯盐酸盐的均相不对称催化氢化 (Scheme 1), 大部分底物以良好的产率、 $>99 / 1$ 的 anti-选择性和较好 到优秀的对映选择性获得一系列手性的 $\beta$-羟基- $\alpha$-氨基 羧酸甲酯化合物. 遗憾的是, 该体系对于烷基取代的反 应底物的催化活性很差, 只获得了中等的对映选择性. 另外, 空气和水分的存在影响该镍催化体系的活性, 因 而需要无水无氧操作, 并在反应体系中加入分子篮除 水. 2009 年, 该课题组 ${ }^{[6]}$ 运用相同的镍催化体系 $\mathrm{Ni}(\mathrm{O}-$ $\mathrm{Ac})_{2} \bullet 4 \mathrm{H}_{2} \mathrm{O} / \mathbf{L 1}$, 在添加剂 $\mathrm{NaBArF}$ 的存在下实现了芳基 取代 $\alpha$-氨基酮盐酸盐的不对称氢化反应, 该反应涉及动 态动力学拆分过程(Scheme 1), 以高产率、>99/1 的 anti选择性和中等到优秀的对映选择性获得手性 $\beta$-氨基醇. 值得注意的是, 添加剂 NaBArF 是必不可少的, 能够显 著提高反应体系的反应活性. 另外, 当使用醋酸作为溶 剂时能够有效地抑制手性底物的消旋化过程, 得到的是
保持手性底物原先构型的氢化产物. 因此，以光学纯的 $\alpha$-氨基酮为底物, 分别运用两种相反构型的配体 $\mathbf{L 1}$ 催 化反应，能分别得到对应的顺式产物和反式产物.

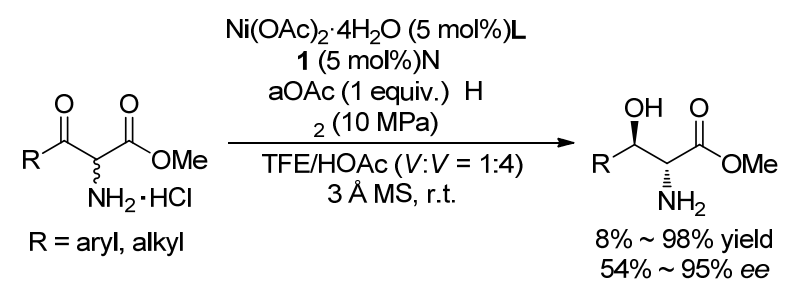<smiles>N[C@H](Cl)C(=O)O</smiles>

$\mathrm{Ni}(\mathrm{OAc})_{2} \cdot 4 \mathrm{H}_{2} \mathrm{O}(10 \mathrm{~mol} \%) \mathrm{L}$ $1(10 \mathrm{~mol} \%) \mathrm{N}$ aOAc (1 equiv.) $\mathrm{N}$ aBArF (10 mol\%) $\mathrm{H}_{2}(10 \mathrm{MPa}), \mathrm{PhMe}$, r.t.
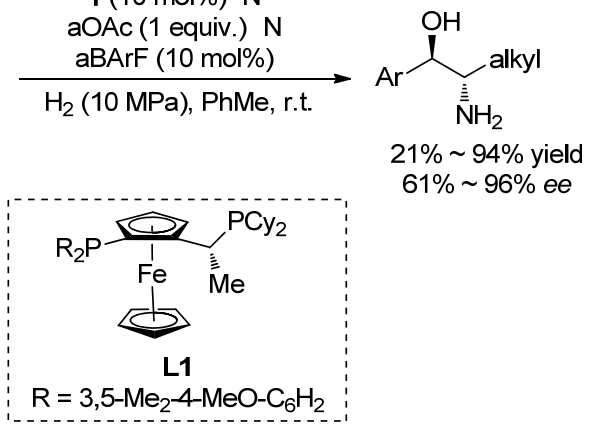

图式 1 镍催化 $\alpha$-氨基- $\beta$-酮酯盐酸盐和取代的芳香 $\alpha$-氨基酮 盐酸盐经过动态动力学拆分过程的不对称氢化反应

Scheme 1 Ni-catalyzed asymmetric hydrogenation of $\alpha$-amino$\beta$-keto ester hydrochlorides and substituted aromatic $\alpha$-aminoketone hydrochlorides through dynamic kinetic resolution

2012 年, 高景星等 ${ }^{[7]}$ 发展了一类新型的 PNO 类型 的手性配体 $\mathbf{L 2}$, 以双(邻甲酰苯基)苯基磷烷和手性 $(R)$ 苯基甘油醇为原料, 经过缩合反应生成手性亚胺后，被 $\mathrm{NaBH}_{4}$ 还原即可得到手性配体 $\mathbf{L} 2$, 合成路线简短高效 (Scheme 2). 随后将其应用于镍催化的简单酮不对称转 移氢化反应中，对于芳基取代的简单酮底物取得了高达 $84 \%$ 的 $e e$ 值, 然而对于脂肪酮底物甲基异丙基酮只获得 了 $10 \%$ 的对映选择性(Scheme 2).

\section{2 镍催化的前手性烯烃( $C=C$ 键)均相不对称 氢化反应}

关于镍催化 $\alpha, \beta$-不饱和烯烃的均相不对称氢化反应 可以追溯到 1992 年, 如 Scheme 3 所示. Corma 等 ${ }^{[8]}$ 发展 了由茮氧羰基保护的 $(L)$-脯氨酸衍生而来的手性氮配体 L3, 并将其应用于 $\mathrm{Rh} 、 \mathrm{Ru} 、 \mathrm{Co}$ 和 $\mathrm{Ni}$ 催化的 $(Z)-2$-苯甲 酰胺基-3-苯基丙烯酸乙酯的不对称氢化反应中, 其中 镍催化体系取得了 $69 \%$ 的 ee 值(Scheme 3).

遗憾的是，在此后相当长的一段时间里镍催化 $\alpha, \beta$ 不饱和烯烃的均相不对称氢化反应没有引起研究者们 的注意. 直到 2014 年, Zhou 课题组 ${ }^{[9]}$ 相继报道了几例 $\alpha, \beta$-不饱和烯烃的镍催化不对称转移氢化反应, 取得了 


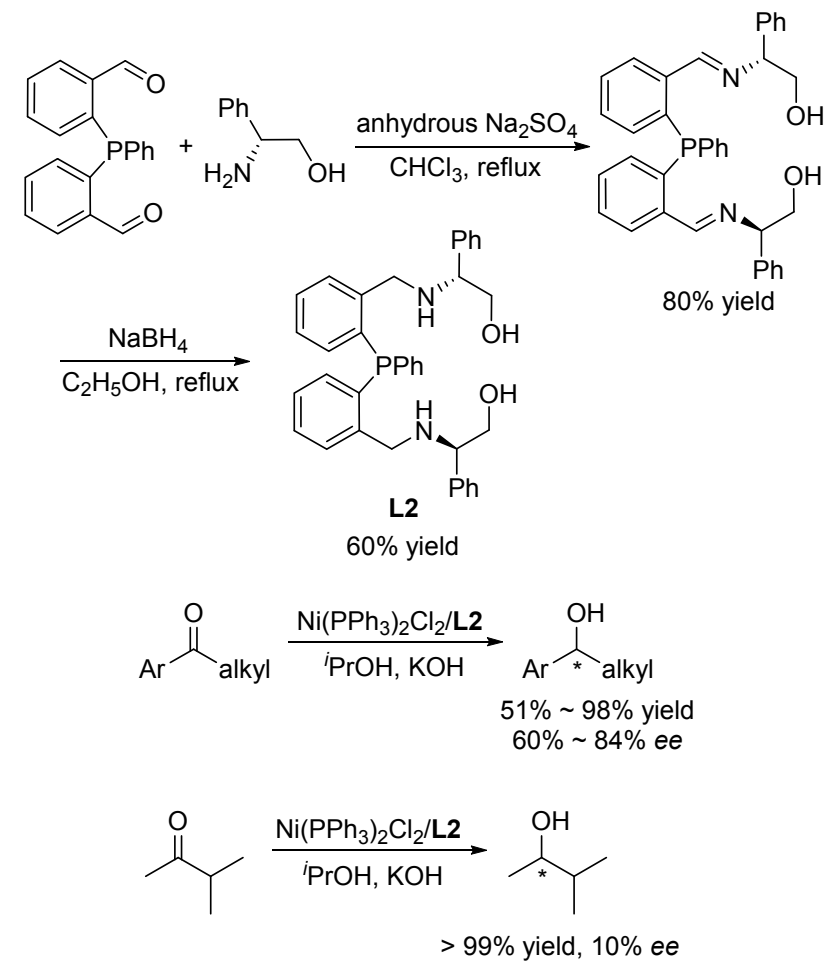

图式 2 PNO 型手性配体在镍催化酮的不对称转移氢化反应 中的应用

Scheme 2 Application of chiral PNO-type ligand in Ni-catalyzed asymmetric transfer hydrogenation of ketones

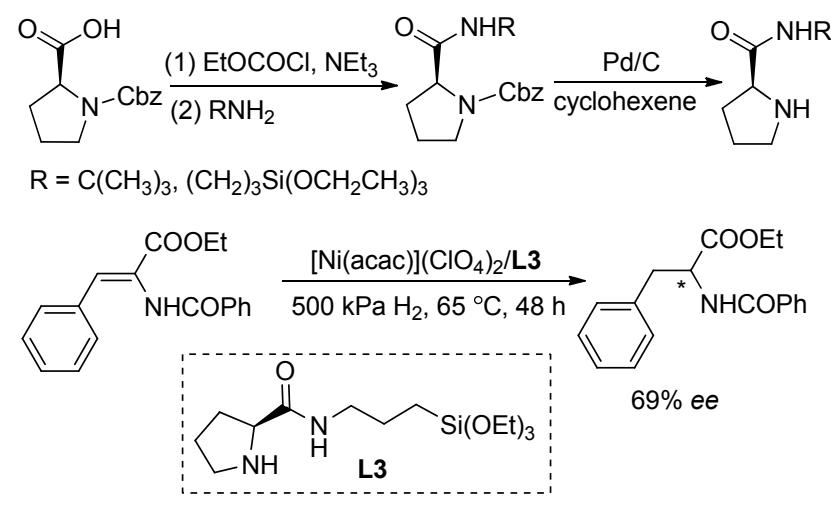

图式 3 镍催化 $(Z)$-2-苯甲酰胺-3-苯丙烯酸乙酯的不对称氢化 反应

Scheme 3 Ni-catalyzed asymmetric hydrogenation of ethyl (Z)-2-benzamido-3-phenylacrylate

非常不错的结果. 他们运用大位阻、强富电性的 $(S)$ Binapine 为配体, 以 $\mathrm{HCOOH} / \mathrm{Et}_{3} \mathrm{~N}$ 作为氢源, 实现了 $\mathrm{Ni}(\mathrm{OAc})_{2} /(S)$-Binapine 催化 $\beta$-脱氢氨基酸酯的不对称转 移氢化反应，取得了 $95 \%$ ～99\%的对映选择性(Scheme 4). 同时, $\alpha$-脱氢氨基酸酯的不对称转移氢化取得了 $86 \% \sim 88 \%$ 的 $e e$ 值. 氛代控制实验结果表明, $\mathrm{HCOOH}$ 与 $\mathrm{Et}_{3} \mathrm{~N}$ 形成的甲酸盐脱羧形成了镍氢活性物种, 对 $\beta$-或 $\alpha-$ 脱氢氨基酸酯底物的 $\beta$ 位发生不对称负氢插入, 生成的 镍烯醇化物种随后被质子化得到产物.

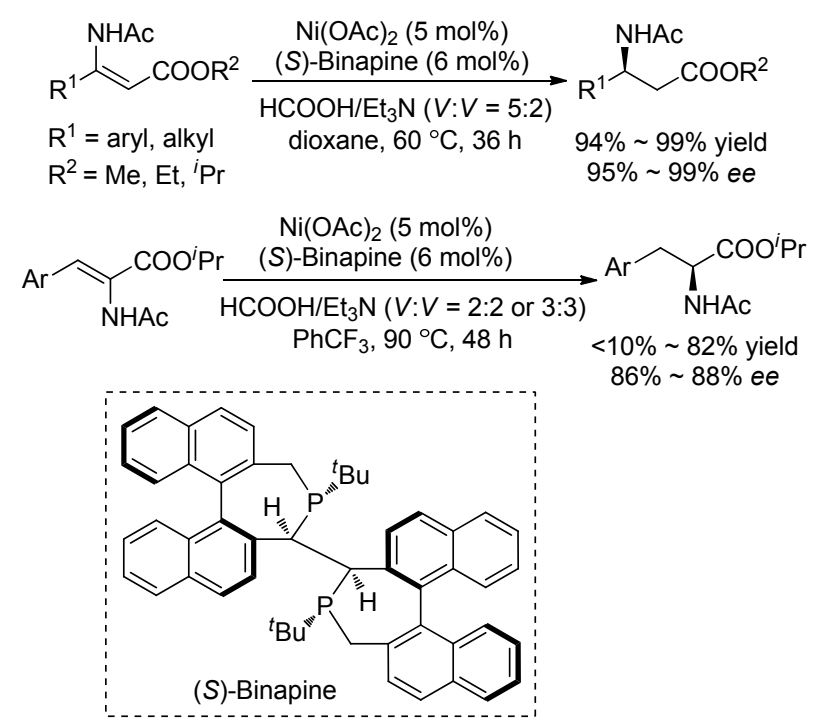

图式 4 镍催化 $\beta$-和 $\alpha$-脱氢氨基酸酯的不对称转移氢化反应 Scheme 4 Ni-catalyzed asymmetric transfer hydrogenation of $\beta$ - and $\alpha$-dehydroamino acid esters

2015 年, 该课题组 ${ }^{[10]}$ 以 $\mathrm{NiBr}_{2}(\mathrm{DME}) /(R, R)-\mathrm{Me}-$ DuPhos 为催化体系, $\mathrm{HCOOH} / \mathrm{Et}_{3} \mathrm{~N}$ (体积比为 $5: 2$ ) 作为 氢源的条件, $80{ }^{\circ} \mathrm{C}$ 下实现了不饱和烯烃的不对称转移氢 化反应。该催化体系底物适用范围比较广, 适用于含羧 酸酯基、酰胺基和氧基的不饱和烯烃类底物, 取得了非 常不错的反应结果, $e e$ 值最高可达 $94 \%$ (Scheme 5).

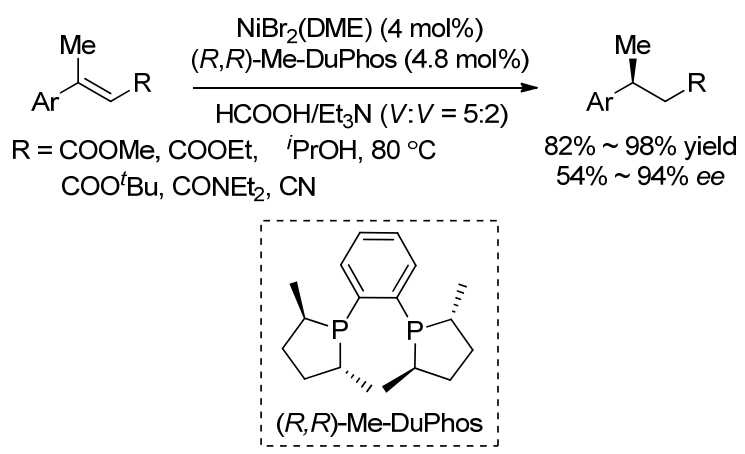

图式 5 镍催化共轭烯烃的不对称转移氢化反应

Scheme 5 Ni-catalyzed asymmetric transfer hydrogenation of conjugated olefins

另外，氝代控制实验结果表明，反应主要途径是甲 酸盐 $\mathbf{A}$ 脱羧生成镍氢活性物种 $\mathbf{B}$, 随后发生不饱和烯烃 底物的插入得到镍烯醇化物 $\mathbf{C}$, 镍烯醇化物 $\mathbf{C}$ 被质子化 释放出氢化产物，同时生成甲酸盐 $\mathbf{A}$, 从而完成一个催 化循环. 催化过程中镍氢活性物种 B 也可能发生去质子 化的可逆平衡, 转化成少量的零价镍 $\left(\mathrm{Ni}^{0}\right)$ 物种 $\mathbf{D}$ (Scheme 6). 另外, $\beta$-甲基对氯肉桂酸甲酯底物还原后得 到脱氯还原产物, 可能是由于发生了 $\mathrm{C}-\mathrm{Cl}$ 键对零价镍 物种的氧化加成所致, 因而从侧面证实了该过程 ${ }^{[10]}$. 


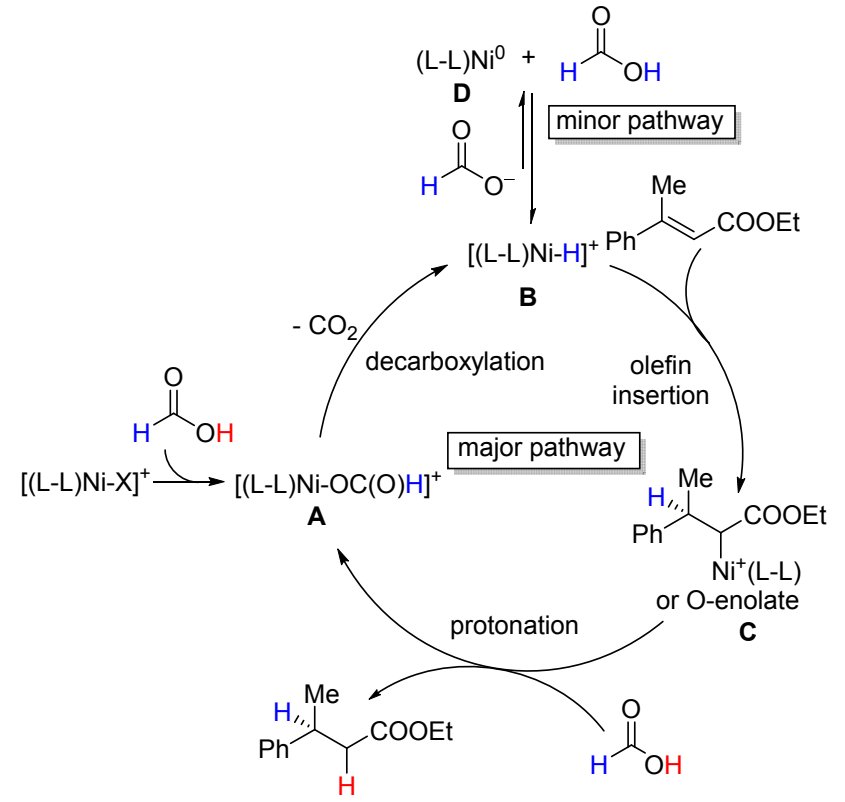

图式 6 镍催化共轭烯烃不对称转移氢化反应的可能机理 Scheme 6 Proposed reaction mechanism for Ni-catalyzed asymmetric transfer hydrogenation of conjugated olefins

2016 年, 该课题组 ${ }^{[11]}$ 运用相同的催化体系 $\mathrm{NiBr}_{2}-$ $(\mathrm{DME}) /(R, R)-\mathrm{Me}-\mathrm{DuPhos}$, 在水和 $\mathrm{AlCl}_{3}$ 存在的条件下, 以 $N, N$-二甲基甲酰胺( DMF) 作为氢源, 水和 $\mathrm{AlCl}_{3}$ 有助 于 DMF 分解生成甲酸根离子, 相当于甲酸的等价类似 物, 成功实现了 $\alpha, \beta$-不饱和羧酸酯的不对称转移氢化, 底物兼容性强, 含有不同吸电子和给电子取代基的苯 基、吡啶基以及噻吩基团的底物都能顺利地进行反应, 以非常高的产率、良好到优秀的对映选择性得到还原产 物(Scheme 7). 然而, 把烯烃上的酯基换为醛基、酮羰 基、酰胺基、氧基和硝基等吸电子基团时, 反应结果不 是很理想, 只能部分转化, 取得中等的对映选择性.

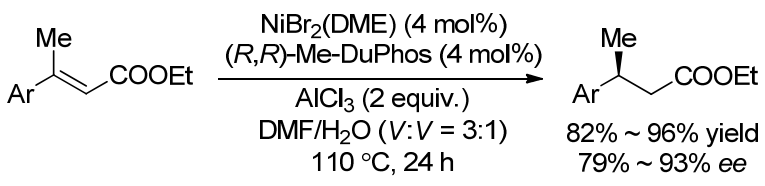

图式 7 以 $N, N$-二甲基甲酰胺(DMF)为氢源的镍催化 $\alpha, \beta$-不饱 和酯的不对称转移氢化反应

Scheme 7 Ni-catalyzed asymmetric transfer hydrogenation of $\alpha, \beta$-unsaturated esters with DMF as hydride source

同年, Chirik 等 ${ }^{[4]}$ 通过高通量实验发现, 以 $\mathrm{H}_{2}$ 作为 氢源, $\mathrm{Bu}_{4} \mathrm{NI}$ 存在的条件下, $\mathrm{Ni}(\mathrm{OAc})_{2} /(S, S)$-Me-DuPhos 体系可以催化前手性 $\alpha, \beta$-不饱和羧酸酯的对映选择性氢 化，取得了良好到优秀的对映选择性(73\% 96\% ee, Scheme 8). 该催化体系适用底物范围很广, 不仅带有吸 电子、给电子取代基苯基的底物能够顺利发生反应, 而 且含有 $\mathrm{N} 、 \mathrm{~S}$ 等易配位原子或者 $\mathrm{Cl}$ 和 $\mathrm{Br}$ 等卤素原子的
底物也能很好地兼容. 由于空间位阻, 苯基邻位有取代 基的底物的反应活性和对映选择性都有所下降.

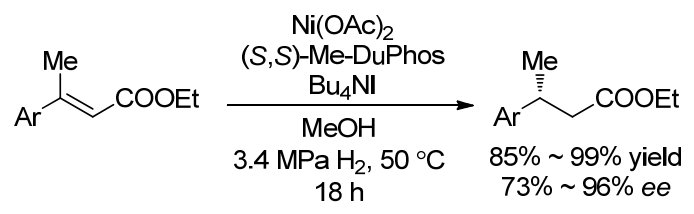

图式 8 镍催化 $\alpha, \beta$-不饱和酯的不对称氢化反应 Scheme 8 Ni-catalyzed asymmetric hydrogenation of $\alpha, \beta$-unsaturated esters

为了揭示该不对称氢化反应的催化机理, 通过连续 变量法和动力学的研究, 表明催化剂的初始状态是 $((S, S)-\mathrm{Me}-\mathrm{DuPhos})_{3} \mathrm{Ni}_{3}(\mathrm{OAc})_{5} \mathrm{I}$ 三金属镍物种, 在反应条 件下转化为活性催化剂物种, 结合 Scheme 9 中相关的 同位素标记实验结果，作者提出了如 Scheme 9 所示的 催化循环机理: 首先在醋酸根配位的协助下, 二价镍物

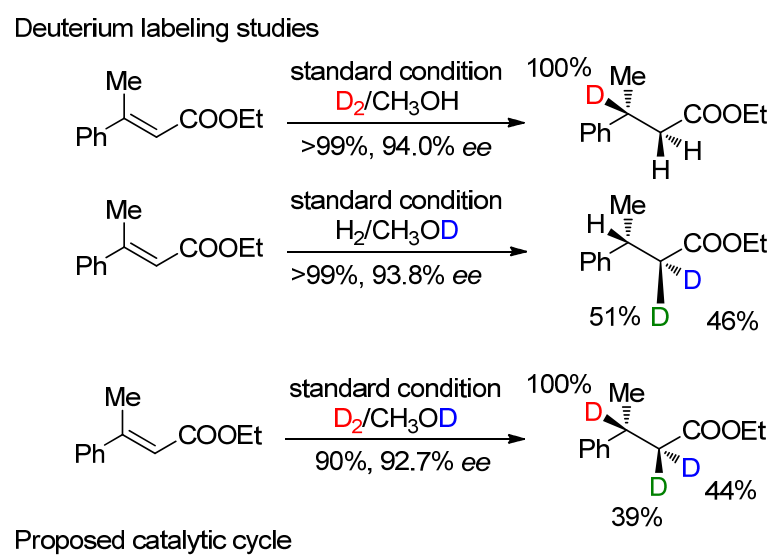

$((\mathrm{S}, \mathrm{S})-\mathrm{Me}-\mathrm{DuPhos})_{3} \mathrm{Ni}_{3}(\mathrm{OAc})_{5} \mathrm{I}$

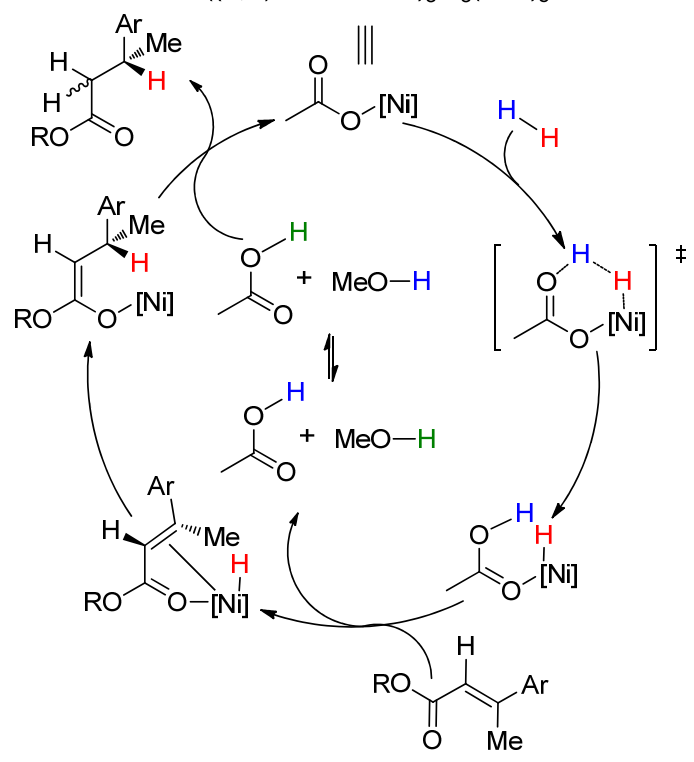

图式 9 気代标记实验研究和可能的催化循环

Scheme 9 Deuterium labeling studies and proposed catalytic cycle 
种活化氢气分子使其发生异裂, 随后底物发生对映选择 性共轭加成生成镍烯醇化物, 然后镍烯醇化物被质子化 并发生互变异构, 释放出产物的同时生成活性催化剂 ${ }^{[4]}$. 该研究工作通过采用多种研究手段深入探索并验证了 镍催化 $\alpha, \beta$-不饱和羧酸酯的均相不对称氢化反应机理, 为后续发展多种共轭烯烃的镍催化不对称氢化反应起 到了重要的指导作用, 是镍催化的均相不对称氢化反应 发展中的重要里程碑.

随后张绪穆等先后将课题组发展的位阻大、刚性强 的双膦配体 $(S)$-Binapine 成功地应用于镍催化的含有硝 基 ${ }^{[12]}$ 、羧酸酯基 ${ }^{[13]}$ 和砜基 ${ }^{[14]}$ 等多种官能团化的不饱和烯 酰胺的不对称氢化反应，以优秀的产率和大部分是 $99 \%$ $e e$ 值及以上的超高对映选择性分别得到手性 $\beta$-氨基硝 基化合物、 $\beta$-氨基酸酯及手性 $\beta$-氨基砜化合物(Scheme 10). 值得强调的是, 硝基烯酰胺的镍催化不对称氢化 的反应条件特别温和, 大部分反应在室温条件下只需 $0.5 \mathrm{MPa}$ 的氢气就能完成. 该反应的同位素标记实验和 密度泛函理论计算(DFT)结果研究表明, 反应机理基本 上类似于 2016 年 Chirik 等提出的不饱和烯烃的共轭加 成机理. 首先, 配位的乙酸根协助二价镍物种活化氢气 分子使其发生异裂, 生成的镍氢活性物种对不饱和烯烃 发生共轭加成，随后发生质子化得到氢化产物。同时， 含酯基和砜基烯酰胺底物的顺式和反式混合物也能顺

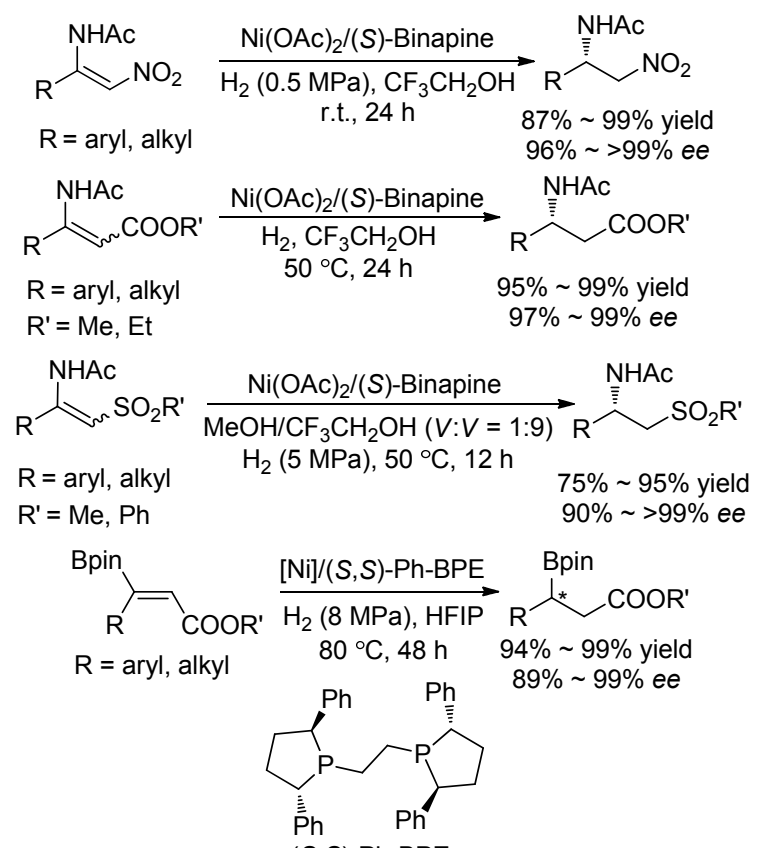

$(S, S)-P h-B P E$

图式 10 镍催化官能团化烯酰胺和不饱和酯的不对称氢化反 应

Scheme 10 Ni-catalyzed asymmetric hydrogenation of functionalized enamides and unsaturated esters
利发生氢化，说明这些烯酰胺的顺式和反式对反应影响 很小. 该催化体系对于官能团化烯酰胺的适用范围之广 及兼容性之宽极大地展现了镍这种廉价金属用于均相 不对称催化氢化的巨大潜力和广阔前景. 需要注意的 是，该催化体系适用的溶剂为甲醇、三氟乙醇和六氟异 丙醇等质子性溶剂, 特别是偏酸性的三氟乙醇和六氟异 丙醇的效果更好. 随后, 该课题组 ${ }^{[15]}$ 运用 $\mathrm{Ni}(\mathrm{OAc})_{2} /$ $(S, S)$-Ph-BPE 催化体系成功地实现了含硼酸频哪醇酯 (Bpin)取代基的不饱和羧酸酯底物的不对称氢化反应, 芳基和烷基取代的底物都能顺利反应，也取得了非常不 错的结果, 而且在低催化剂用量下的克级规模不对称氢 化反应能够顺利完成.

另外, 2019 年该课题组 ${ }^{[16]}$ 实现了镍催化具挑战性的 四取代氟代烯酰胺酯的不对称催化氢化, 以高产率、高 非对映选择性和优异的对映选择性得到了顺式的手性 $\alpha$-氟代- $\beta$-氨基酸酯，反应体系中没有脱氟的副产物 (Scheme 11). 基于同位素気代实验结果以及超高的非对 映选择性 $(d r>99 / 1)$, 作者认为反应溶剂的酸性对于非 对映选择性的控制起着关键的作用，偏酸性的三氟乙醇 作为反应溶剂有利于加速镍氢活性物种对 $\mathrm{C}=\mathrm{C}$ 键的加 成中间体快速发生质子解，有效地抑制了加成中间体向 镍烯醇化物的转化, 从而只得到单一的顺式氢化产物.

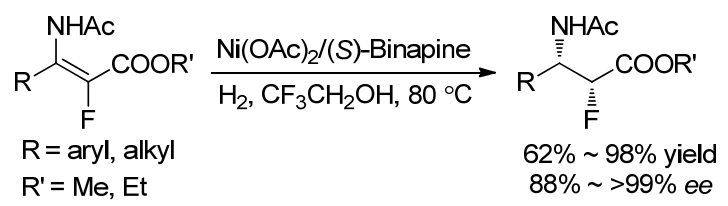

图式 11 镍催化四取代氟代的烯酰胺不对称氢化反应 Scheme 11 Ni-catalyzed asymmetric hydrogenation of tetrasubstituted fluorinated enamides

最近, 该课题组 ${ }^{[17]}$ 报道了 $\mathrm{Ni}(\mathrm{OTf})_{2} /(S, S)$-Ph-BPE 催 化对称型 $\gamma, \gamma$ 二取代环已二烯酮的去对称化不对称氢化 反应，以优秀的产率得到 $\gamma$ 位含手性季碳中心的环己烯 酮 (92\% 99\% ee) 和螺环环己烯酮 (96\% 99\%ee) (Scheme 12). 值得关注的是, 该催化体系与之前相比有 所变化, 采用了 $\mathrm{Ni}(\mathrm{OTf})_{2}$ 作为镍源, 使用了非质子型溶 剂甲苯替代了以往最常用的甲醇、三氟乙醇和六氟异丙 醇这三种质子型溶剂. 之前的 $\mathrm{Ni}(\mathrm{OAc})_{2}$ 镍源体系使用 甲苯等非质子溶剂, 一般是不发生反应或者只发生微量 反应.

\section{3 镍催化的前手性亚胺 $(C=N$ 键)均相不对称 氢化反应}

简单亚胺的催化氢化极具挑战性主要是由于其反 应活性比较低和还原产物胺类化合物容易与金属中心 

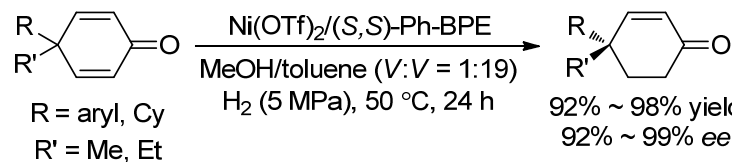

$\mathrm{R}^{\prime}=\mathrm{Me}, \mathrm{Et}$

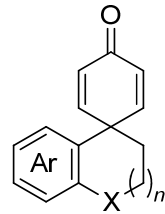

$\mathrm{X}=\mathrm{CH}_{2}, \mathrm{O}, \mathrm{S}$

$n=0,1,2$

图式 12 镍催化 $\gamma, \gamma$-二取代环己二烯酮的不对称氢化反应

Scheme 12 Ni-catalyzed desymmetric hydrogenation of $\gamma, \gamma$ disubstituted cyclohexadienones

配位使催化剂失活. 镍催化氢化体系相对于贵重金属体 系来说催化活性总体上偏低, 因此镍直接催化不对称氢 化简单亚胺是比较困难和具有挑战性的. 目前, 镍催化 不对称氢化亚胺的 $\mathrm{C}=\mathrm{N}$ 键的策略通常是：通过 $\mathrm{N}$ 上连 有磺酰基、磷酰基等基团来活化 $\mathrm{C}=\mathrm{N}$ 双键使其易于发 生还原反应, 主要是磺酰亚胺、磷酰亚胺和腙类等高度 活化的亚胺, 反应机理类似于前面总结的镍-氢催化共 轭加成还原机理.

2015 年, Zhou 等 ${ }^{[18]}$ 通过利用位阻大、富电性强的配 体 $(S)$-Binapine 组成 $\left[\mathrm{NiCl}_{2}(\mathrm{DME})\right] /(S)$-Binapine 催化体 系, 在 $\mathrm{HCOOH} / \mathrm{Et}_{3} \mathrm{~N}$ 提供氢源的条件下，成功实现了苯 甲酰基、乙酰基和苯基活化的朋类亚胺的高对映选择性 转移氢化反应, $e e$ 值高达 $98 \%$ (Scheme 13). 根据同位素 氞代实验结果，作者提出甲酸脱羧提供甲酰基的 $\mathrm{H}$ 作为 负氢，生成镍-氢活性物种对活化亚胺共轭加成，随后 质子解和互变异构得到氢化产物的机理. 通过 DFT 计 算, 作者发现在过渡态的结构中, 过渡态 TS- $R$ 形成的 凹面比过渡态 TS- $S$ 的凹面与底物更能互补, 同时配体 (S)-Binapine 叔丁基上的 $\mathrm{CH}$ 与肼类底物的氧原子存在 弱的相互作用，正是催化剂与底物形状的互补和存在的 弱相互作用导致了该不对称转移氢化的高立体选择性.

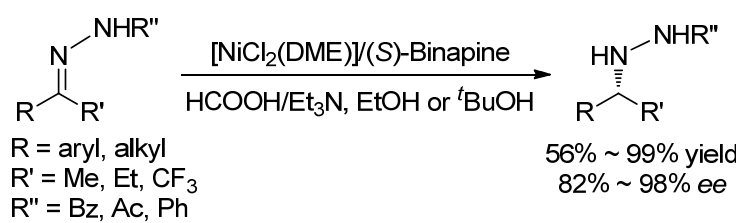

图式 13 镍催化的腙不对称转移氢化反应

Scheme 13 Ni-catalyzed asymmetric transfer hydrogenation of hydrazones

相对于酮亚胺的还原氢化反应, 酮的还原胺化能够 有效地规避亚胺类化合物不稳定、难以分离提纯的问题, 是高效构建手性胺化合物的有效途径之一. 2016 年, Zhou 等 ${ }^{[19]}$ 发展了以芳香胺和苯甲酰朋为胺源, 以 HCO-
$\mathrm{OH} / \mathrm{Et}_{3} \mathrm{~N}$ 作为氢源，在分子篮作为脱水剂的条件下, $\mathrm{Ni}(\mathrm{OTf})_{2} /(R, R)-\mathrm{Ph}-\mathrm{BPE}$ 或 $(S)$-Binapine 催化成功地实现 了苯乙酮类底物的不对称还原胺化，以良好到优秀的高 对映选择性得到芳基和苯甲酰基保护的手性胺类化合 物(Scheme 14). 不同保护基胺源的还原胺化可以通过 使用不同的配体和 $\mathrm{Ni}(\mathrm{OTf})_{2}$ 组成最优的催化体系. 在 $\mathrm{Ni}(\mathrm{OTf})_{2} /(R, R)-\mathrm{Ph}-\mathrm{BPE}$ 催化苯乙酮类底物和芳香胺的转 移还原胺化中, 芳香胺上的取代基对反应立体选择性的 影响很大，当使用 3,5-二甲基 4-甲氧基苯胺作为胺源时 能够获得最高的对映选择性. 对于烷基酮底物的转移还 原胺化，使用苯甲酰肼为胺源能得到 86\% 90\%的对映 选择性. 而且, 使用 1,2-二氨基苯和 $\alpha$-酮醛发生缩合反 应生成喹喔啉分子, 不需要分离, 在 $\mathrm{Ni} /(S)$ - TangPhos 作 为催化剂的条件下发生双还原胺化反应直接生成手性 的四氢喹喔啉, $\mathrm{C}(2)$ 上取代基为甲基时能够获得 $93 \% e e$, 为苯基时只能获得中等的对映选择性( $72 \% e e)$, 而且添 加剂 $n-\mathrm{Bu}_{4} \mathrm{NI}$ 能够改善对映选择性并减少 $N$-甲酰化副反 应.
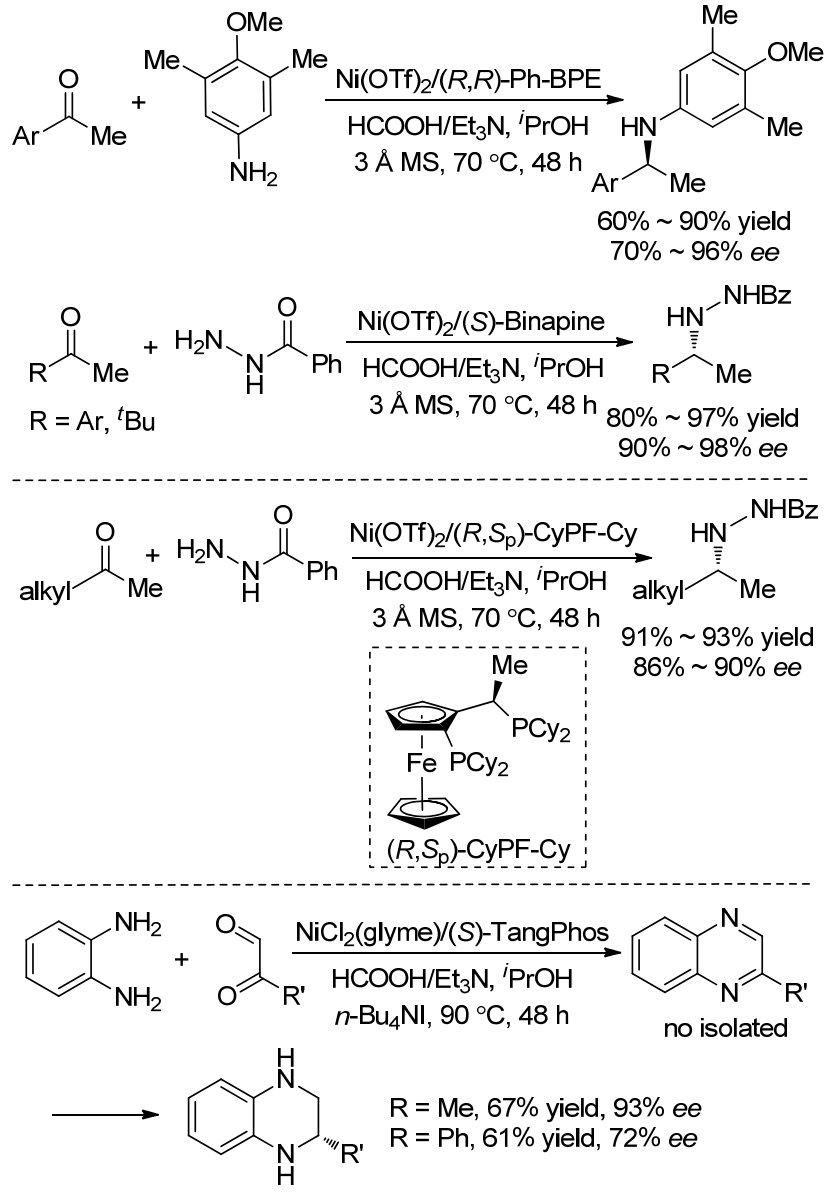

图式 14 镍催化芳胺、苯肼与酮的不对称还原胺化反应 Scheme 14 Ni-catalyzed asymmetric reductive amination of ketones using both arylamines and benzhydrazide 
作者 ${ }^{[19]}$ 通过理论计算离子型镍氢活性物种对 $N$-芳 基亚胺发生插入反应时的过渡态发现, 生成 $(R)$-isomer 的过渡态 TS- $R$ 的能垒比生成 $(S)$-isomer 的过渡态 TS- $S$ 的能垒高出 $8.8 \mathrm{~kJ} / \mathrm{mol}$, 与实际测得生成产物的 $76 \%$ ee 值吻合(Scheme 15).

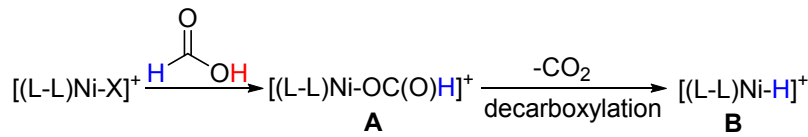

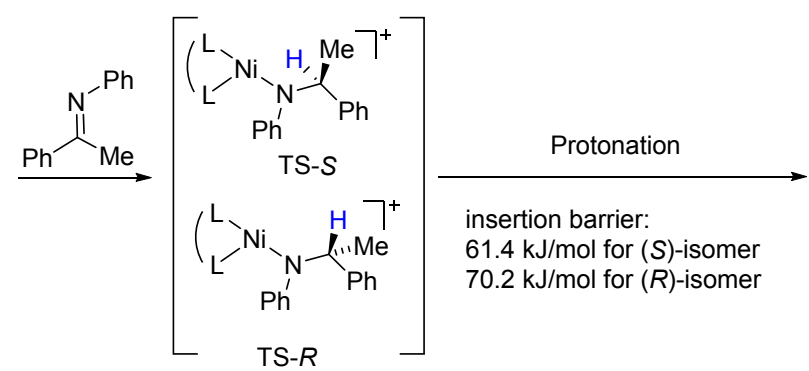<smiles>CC(C)C(=O)Nc1ccccc1</smiles>

(S)-isomer

$76 \%$ ee

图式 15 镍催化芳胺与酮的不对称还原胺化反应的计算研究 Scheme 15 Calculations on Ni-catalyzed asymmetric reductive amination of ketones with aryl amines

2019 年, Zhou 课题组 ${ }^{[20]}$ 再次报道了一例关于酮分 步进行的转移还原胺化, 使用更具挑战性的、亲核性更 弱的磺酰胺和二苯基磷酰胺作为胺源, 实现了包括简单 芳基酮、环状苯并酮和二芳基甲酮等各类酮的不对称转 移还原胺化, 大部分取得了大于 $90 \%$ ee 值的高对映选 择性(Scheme 16). 需要注意的是, 该转移还原胺化是分 步进行的, 同类底物先与磺酰胺或二苯基磷酰胺在 $\mathrm{Ti}(\mathrm{OEt})_{4}$ 存在的条件下于甲苯中回流制备成亚胺类化合 物, 然后直接进行下一步的转移还原胺化, 中间无需分 离提纯亚胺类化合物, 巧妙地解决了该类不稳定亚胺提 纯困难的的问题.

紧接着，张万斌等 ${ }^{[21]}$ 报道了利用 $\mathrm{Ni}(\mathrm{OAc})_{2} \cdot \mathrm{H}_{2} \mathrm{O} /$ $(R, R)$-QuinoxP*体系实现链状酮和苯并酮的叔丁基磺酰 酮亚胺的高对映选择性氢化, 以中等到优秀的 $e e$ 值得 到一系列手性叔丁基磺酰胺产物(Scheme 17). 芳环上 对位和间位含吸电子取代基的苯乙酮类型叔丁基磺酰 亚胺底物发生不对称氢化反应, 得到的产物的收率和对 映选择性都有所下降. 对于苯并磺酰亚胺类底物的不对 称氢化, 底物和催化剂用量的比例能达到 10500 , 是目 前已报道镍催化体系所能取得的最高值, 是镍催化均相 不对称氢化反应的重大突破, 使其离在工业上的应用更 近一步.

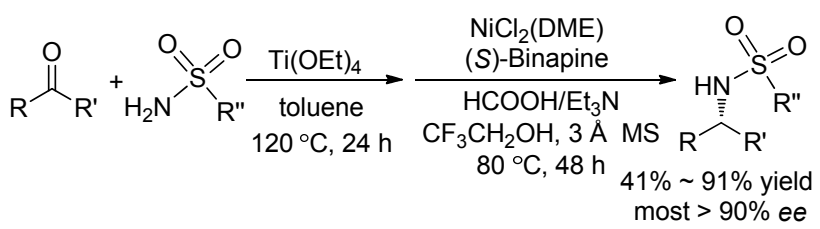

$\mathrm{R}=$ aryl, alkyl (different bisphosphines as ligand respectively); $\mathrm{R}^{\prime}=\mathrm{Me}$, aryl; $\mathrm{R}^{\prime \prime}=$ aryl, alkyl
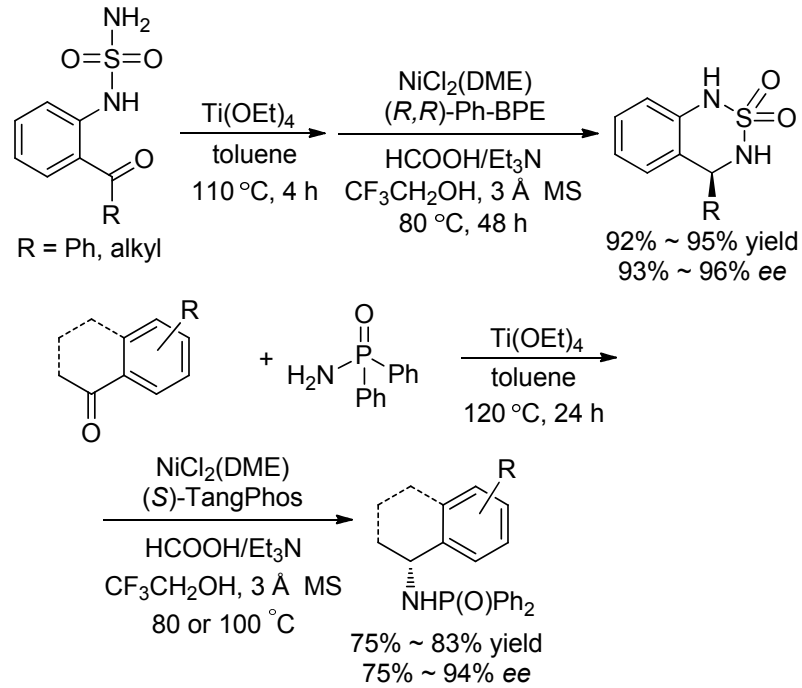

图式 16 镍催化酮的分步不对称还原胺化反应

Scheme 16 Ni-catalyzed asymmetric stepwise reductive amination of ketones

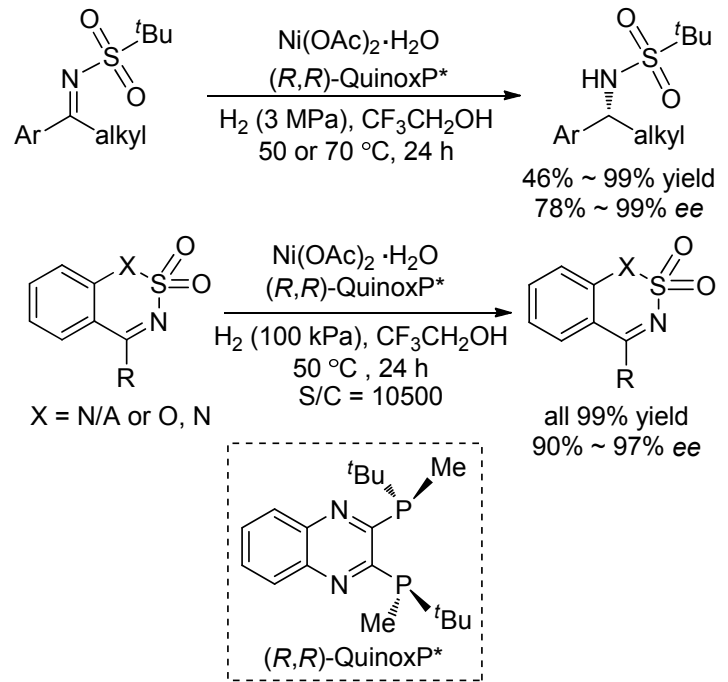

图式 17 镍催化 $N$-磺酰亚胺的不对称氢化反应

Scheme 17 Ni-catalyzed asymmetric hydrogenation of $\mathrm{N}$-sulfonyl imines

进一步的研究发现，在镍盐和配体的络合物之间存 在如 Scheme 18 所示的配位平衡, 无活性的二聚体络合 物 a 在添加的过量镍盐作用下转化成活性催化络合物 $\mathbf{a}^{\prime}$ ，从而提高了催化效率. 作者也通过理论计算来验证 反应催化机理, 发现生成 $R$ 构型产物的过渡态 TS- $R$ 能 垒比生成 $S$ 构型产物的过渡态 TS- $S$ 能垒低 $20.9 \mathrm{~kJ} / \mathrm{mol}$, 
认为反应是通过镍-氢活性物种直接加成进攻亚胺 $\mathrm{C}=$ $\mathrm{N}$ 双键，过渡态 TS- $R$ 中催化剂和底物之间存在着的许 多弱相互作用, 能稳定该过渡态, 有利于提高该催化体 系的催化活性.

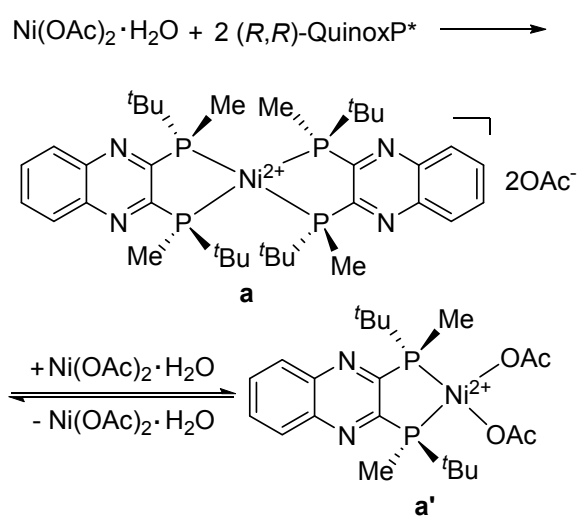

图式 18 镍盐与其络合物的配位平衡

Scheme 18 Coordination equilibrium exists between the nickel salt and its complex

张绪穆课题组在致力于发展镍催化共轭烯烃的不 对称氢化反应的同时，也陆续发展了镍催化磺酰基活化 亚胺的不对称氢化反应, 取得了不错的结果. 几乎与张 万斌课题组同时, 张绪穆等 ${ }^{[22]}$ 利用 $\mathrm{Ni}(\mathrm{OAc})_{2} /(S, S)-\mathrm{Ph}-$ BPE 体系成功地实现了环状磺酰亚胺酯的不对称氢化 反应, 以高产率和高对映选择性获得光学纯的环状磺酰 胺酯产物( $83 \% \sim>99 \% e e$, Scheme 19). 该类手性环状 磺酰胺酯是十分重要的合成中间体, 可以进一步转化成 手性胺、手性氨基醇和手性噁唑啉等配体. 该催化体系 兼容各种底物, 不论是含不同电性取代基的芳基底物还 是简单烷基底物, 反应都能很好地进行. 该催化体系催 化的克级规模反应能够顺利进行, 对映选择性几乎保持 不变, 高收率地得到氢化产物. 另外, 由苯乙酮和苯并 环状酮衍生而来的磺酰基亚胺也适用于该催化体系, 反 应完全并取得 97\% >99\%的对映选择性.<smiles>[R]C1=NS(=O)(=O)OC1</smiles>

$\mathrm{R}=$ aryl, alkyl<smiles>[N-]=C1CCc2ccccc21</smiles>

$\mathrm{Ni}(\mathrm{OAc})_{2} /(S, S)-\mathrm{Ph}-\mathrm{BPE}$

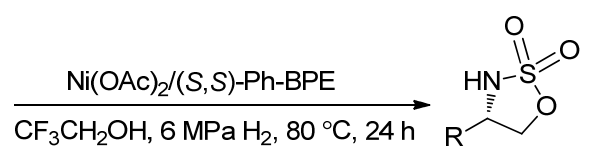

$55 \% \sim 99 \%$ yield $83 \% \sim>99 \%$ ee

$\mathrm{CF}_{3} \mathrm{CH}_{2} \mathrm{OH}, 6 \mathrm{MPa} \mathrm{H}, 80^{\circ} \mathrm{C}, 24 \mathrm{~h}$

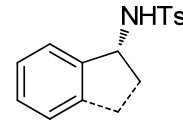

$96 \% \sim 97 \%$ yield $97 \%$ $99 \%$ ee

图式 19 镍催化 $N$-磺酰亚胺的不对称氢化反应 Scheme 19 Ni-catalyzed asymmetric hydrogenation of cyclic sulfamidate imines

作者进行的同位素标记实验研究表明, 当用 $\mathrm{D}_{2}$ 代
替 $\mathrm{H}_{2}$ 进行反应时，芐基 $\mathrm{H}$ 被完全気代，氨基上的活泼氢 发生部分 $\mathrm{H} / \mathrm{D}$ 交换, 说明芐基 $\mathrm{H}$ 来自 $\mathrm{H}_{2}$ 异裂产生的负 氢; 当溶剂换为氞代的 $\mathrm{CF}_{3} \mathrm{CH}_{2} \mathrm{OD}$ 时, 只有磺酰胺上的 $\mathrm{H}$ 被部分気代; 正常的氢化产物以相同的反应条件在 $\mathrm{CF}_{3} \mathrm{CH}_{2} \mathrm{OD}$ 中搅拌, 只有磺酰胺上的 $\mathrm{H}$ 被部分気代 (Scheme 20) ${ }^{[22]}$. 结合该实验结果和前人的相关工作, 作 者提出了类似于 2016 年 Chirik 等提出的镍催化共轭加 成机理: 先是镍在配位的醋酸根离子的协助下活化异裂 $\mathrm{H}_{2}$ 分子生成的镍-氢活性物种对磺酰亚胺底物发生共轭 加成, 然后质子解释放出产物的同时循环恢复催化剂.

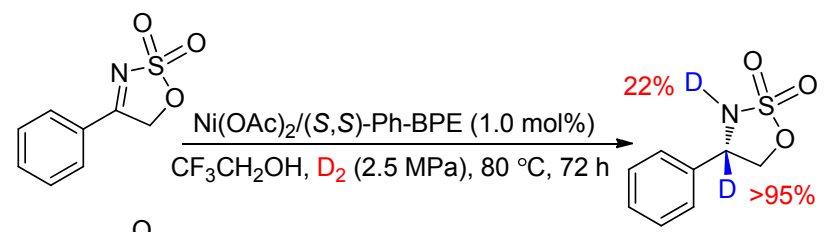

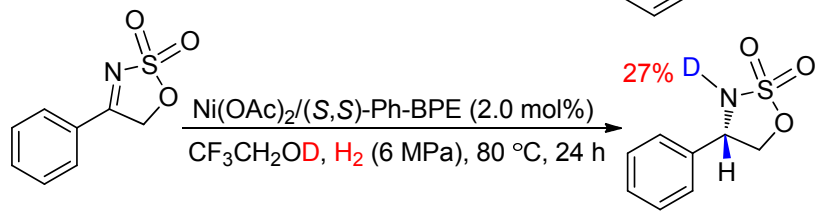<smiles>[2H]N1[C@H](c2ccccc2)COS1(=O)=O</smiles>

图式 20 镍催化 $N$-磺酰亚胺的不对称氢化反应的氛标记实验 研究

Scheme 20 Deuterium-labeling experiments of cyclic sulfamidate imines

最近该课题组 ${ }^{[23]}$ 在前述工作的基础上顺利实现了 $\mathrm{Ni}(\mathrm{OAc})_{2} /(S, S)-\mathrm{Ph}-\mathrm{BPE}$ 催化 $\alpha, \beta$-不饱和磺酰亚胺的化学 选择性氢化反应, 高化学选择性和高对映选择性地获得 了一系列保留烯烃 $\mathrm{C}=\mathrm{C}$ 双键的手性烯丙基胺化合物 $(90 \% \sim>99 \% e e$, Scheme 21). 需要注意的是, 烯烃的 端位必须带有两个取代基，对于烯烃端位单取代的 $\alpha, \beta$ 不饱和磺酰亚胺会生成烯烃 $\mathrm{C}=\mathrm{C}$ 双键也被同时还原的 氢化产物。

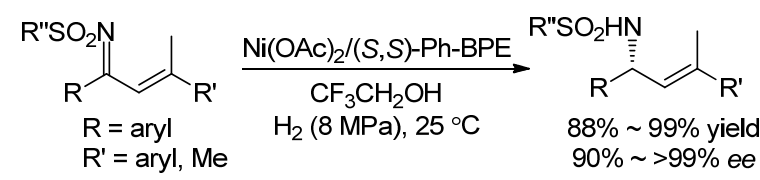

图式 21 镍催化 $\alpha, \beta$-不饱和 $N$-磺酰亚胺的不对称氢化反应 Scheme 21 Ni-catalyzed asymmetric hydrogenation of $\alpha, \beta$ unsaturated sulfonyl imines

\section{4 总结与展望}

自 2016 年 Chirik 等提出了镍催化氢化不饱和烯烃 的共轭加成机理后，镍催化的均相不对称氢化反应取得 了一些非常不错的成果, 推动了该研究领域的发展, 但 
总体上还是处于起步的阶段. 目前已发展的镍催化体系 主要使用富电性的双膦配体, 相对于贵重金属催化的不 对称氢化反应中使用的含 $\mathrm{P} 、 \mathrm{~N}$ 和 $\mathrm{S}$ 等多种类型的配体 来说, 催化体系相对比较单一, 而且催化活性普遍偏低, 使得镍催化体系在现阶段很难替代贵重金属催化体系 应用于工业化的不对称氢化反应. 虽然 2016 年提出的 不饱和烯烃的镍催化共轭加成机理取得了进展, 但是镍 催化酮的不对称均相氢化机理尚不清晰. 因此, 需要进 一步深入研究和挖掘镍催化不对称氢化反应可能存在 的其他反应机理, 总结催化氢化的反应途径和反应机 理, 从而为镍催化不对称氢化反应设计更为高效和易得 的新型手性配体和发掘新的催化体系提供新思路, 尽早 发展出高效、高选择性的通用型镍催化体系, 在提高催 化活性的同时扩大适用底物范围, 实现镍催化体系对于 简单烯烃、未活化亚胺、各种酮以及含多种不饱和键底 物的高活性、高选择性的不对称氢化反应, 从而发展新 颖、高效的催化体系和不对称催化氢化新反应仍然是本 领域的研究重心. 因此, 可以预计今后镍催化的不对称 催化氢化反应研究将会有更多的突破, 使其成为催化不 对称合成手性化合物的原子经济、环境友好方法之一.

\section{References}

[1] (a) Noyori, R.; Takaya, H. Acc. Chem. Res. 1990, 23, 345.

(b) Tang, W.; Zhang, X. Chem. Rev. 2003, 103, 3029.

(c) Minnaard, A. J.; Feringa, B. L.; Lefort, L.; de Vries, J. G. Acc. Chem. Res. 2007, 40, 1267.

(d) Zhang, W.; Chi, Y.; Zhang, X. Acc. Chem. Res. 2007, 40, 1278.

(e) Zhou, Y.-G. Acc. Chem. Res. 2007, 40, 1357.

(f) Roseblade, S. J.; Pfaltz, A. Acc. Chem. Res. 2007, 40, 1402

(g) Xie, J. H.; Zhu, S. F.; Zhou, Q. L. Chem. Rev. 2011, 111, 1713.

(h) Wang, Y.; Zhang, Z.; Zhang, W. Chin. J. Org. Chem. 2015, 35, 528 (in Chinese).

(王英杰, 张振锋, 张万斌, 有机化学, 2015, 35, 528.)

(i) Wang, D.-S.; Chen, Q.-A.; Lu, S.-M.; Zhou, Y.-G. Chem. Rev. 2012, 112, 2557.

(j) Chen, Q.-A.; Ye, Z.-S.; Duan, Y.; Zhou, Y.-G. Chem. Soc. Rev. 2013, 42, 497 .

(k) Verendel, J. J.; Pamies, O.; Dieguez, M.; Andersson, P. G. Chem. Rev. 2014, 114, 2130

(1) Zhang, Z.; Butt, N. A.; Zhang, W. Chem. Rev. 2016, 116, 14769. (m) de Vries, J. G.; Elsevier, C. J. Handbook of Homogeneous Hydrogenation, Wiley-VCH, Weinheim, Germany, 2007.

(n) Ma, Y.; Zhang, Y. J.; Zhang, W. Chin. J. Org. Chem. 2007, 27, 289 (in Chinese).

(马元辉, 张勇健, 张万斌, 有机化学, 2007, 27, 289.)

(o) Xie, J.-H.; Zhou, Q.-L. Acta Chim. Sinica 2012, 70, 1427 (in Chinese).

(谢建华, 周其林, 化学学报, 2012, 70, 1427.)

(p) Wang, Z.; Zhang, Z.; Liu, Y.; Zhang, W. Chin. J. Org. Chem. 2016, 36, 447 (in Chinese).

(王志惠, 张振锋, 刘燕刚, 张万斌, 有机化学, 2016, 36, 447.)

(q) Chen, S.; Yang, W.; Yao, Y.; Yang, X.; Deng, Y.; Yang, D. Chin.
J. Org. Chem. 2018, 38, 2534 (in Chinese).

(陈姝琪，杨文，姚永祺，杨新，邓颖颖，杨定乔，有机化学， 2018, 38, 2534.)

(r) Liang, Z.; Yang, T.; Gu, G.; Dang, L.; Zhang, X. Chin. J. Chem. 2018, 36, 851 .

[2] (a) Morris, R. H. Chem. Soc. Rev. 2009, 38, 2282.

(b) Morris, R. H. Acc. Chem. Res. 2015, 48, 1494

(c) Chirik, P. J. Acc. Chem. Res. 2015, 48, 1687

(d) Li, Y.-Y.; Yu, S.-L.; Shen, W.-Y.; Gao, J.-X. Acc. Chem. Res. 2015, 48, 2587.

(e) Harman, W. H.; Peters, J. C. Chem. Rev. 2015, 115, 3170.

(f) Zhang, Z.; Butt, N. A.; Zhou, M.; Liu, D.; Zhang, W. Chin. J. Chem. 2018, 36, 443.

[3] (a) Tai, A. and Sugimura, T. In Chiral Catalysts Immobilization and Recycling, Eds.: De Vos, D. E.; Vankelecom, I. F. J.; Jacobs, P. A., Wiley-VCH, Weinheim, 2000.

(b) Sarko, C. R.; DiMare, M.; Yus, M.; Alonso, F. e-EROS Encyclopedia of Reagents for Organic Synthesis, Wiley-VCH, Weinheim 2014.

(c) Angulo, I. M.; Kluwer, A. M.; Bouwman, E. Chem. Commun. 1998, 2689 .

(d) Angulo, I. M.; Bouwman, E.; van Gorkum, R.; Lok, S. M.; Lutz, M.; Spek, A. L. J. Mol. Catal. A: Chem. 2003, 202, 97.

(e) Harman, W. H.; Peters, J. C. J. Am. Chem. Soc. 2012, 134, 5080. (f) Cammarota, R. C.; Lu, C. C. J. Am. Chem. Soc. 2015, 137, 12486.

[4] Shevlin, M.; Friedfeld, M. R.; Sheng, H.; Pierson, N. A.; Hoyt, J. M.; Campeau, L.; Chirik, P. J. J. Am. Chem. Soc. 2016, 138, 3562.

[5] Hamada, Y.; Koseki, Y.; Fujii, T.; Maeda, T.; Hibino, T.; Makino, K. Chem. Cотmun. 2008, 6206.

[6] Hibino, T.; Makino, K.; Sugiyama, T.; Hamada, Y. ChemCatChem 2009, 1,237

[7] Dong, Z. R.; Li, Y. Y.; Yu, S. L.; Sun, G. S.; Gao, J. X. Chin. Chem Lett. 2012, 23, 533.

[8] Corma, A.; Iglesias, M.; Del Pino, C.; Sanchez, F. J. Organomet Chem. 1992, 431, 233.

[9] Yang, P.; Xu, H.; Zhou, J. Angew. Chem., Int. Ed. 2014, 53, 12210.

[10] Guo, S. Y.; Yang, P.; Zhou, J. Chem. Commun. 2015, 51, 12115.

[11] Guo, S.; Zhou, J. Org. Lett. 2016, 18, 5344.

[12] Gao, W.; Lv, H.; Zhang, T.; Yang, Y.; Chung, L. W.; Wu, Y.; Zhang, X. Chem. Sci. 2017, 8, 6419

[13] Li, X.; You, C.; Li, S.; Lv, H.; Zhang, X. Org. Lett. 2017, 19, 5130.

[14] Long, J.; Gao, W.; Guan, Y.; Lv, H.; Zhang, X. Org. Lett. 2018, 20 , 5914.

[15] Han, Z.; Liu, G.; Zhang, X.; Li, A.; Dong, X.-Q.; Zhang, X. Org. Lett. 2019, 21, 3923.

[16] Guan, Y.; Han, Z.; Li, X.; You, C.; Tan, X.; Lv, H.; Zhang, X. Chem. Sci. 2019, 10, 252.

[17] You, C.; Li, X.; Gong, Q.; Wen, J.; Zhang, X. J. Am. Chem. Soc. 2019, 141, 14560 .

[18] Xu, H.; Yang, P.; Chuanprasit, P.; Hirao, H.; Zhou, J. Angew. Chem., Int. Ed. 2015, 54, 5112.

[19] Yang, P.; Lim, L. H.; Chuanprasit, P.; Hirao, H.; Zhou, J. Angew. Chem., Int. Ed. 2016, 55, 12083.

[20] Zhao, X.; Xu, H.; Huang, X.; Zhou, J. Angew. Chem., Int. Ed. 2019, $58,292$.

[21] Li, B.; Chen, J.; Zhang, Z.; Gridnev, I. D.; Zhang, W. Angew. Chem., Int. Ed. 2019, 58, 7329 .

[22] Liu, Y.; Yi, Z.; Tan, X.; Dong, X.-Q.; Zhang, X. iScience 2019, 19, 63.

[23] Zhao, X.; Zhang, F.; Liu, K.; Zhang, X.; Lv, H. Org. Lett. 2019, 21, 8966. 